\title{
Robotic Recurrent Hiatal Hernia Repair, Collis Gastroplasty, and Nissen Fundoplication
}

Salim Hosein, MD

Department of Surgery, University of Nebraska Medical Center, Omaha, Nebraska.

Center for Advanced Surgical Technology, University of Nebraska Medical Center, Omaha, Nebraska. Current address: Department of General Surgery, Tulane Medical Center, New Orleans, Louisiana.

\section{Dietric Hennings, MD}

Department of Surgery, University of Nebraska Medical Center, Omaha, Nebraska.

Center for Advanced Surgical Technology, University of Nebraska Medical Center, Omaha, Nebraska. Current address: Department of General Surgery, Tulane Medical Center, New Orleans, Louisiana.

\section{Crystal Krause, PhD}

Center for Advanced Surgical Technology, University of Nebraska Medical Center, Omaha, Nebraska.

\section{Dmitry Oleynikov, MD}

Department of Surgery, University of Nebraska Medical Center, Omaha, Nebraska.

Center for Advanced Surgical Technology, University of Nebraska Medical Center, Omaha, Nebraska. E-mail: doleynik@unmc.edu

This video was first presented as a video abstract at the American College of Surgeons 2018 Clinical Congress.

(c) Mary Ann Liebert, Inc. DOI: 10.1089/vor.2019.0604
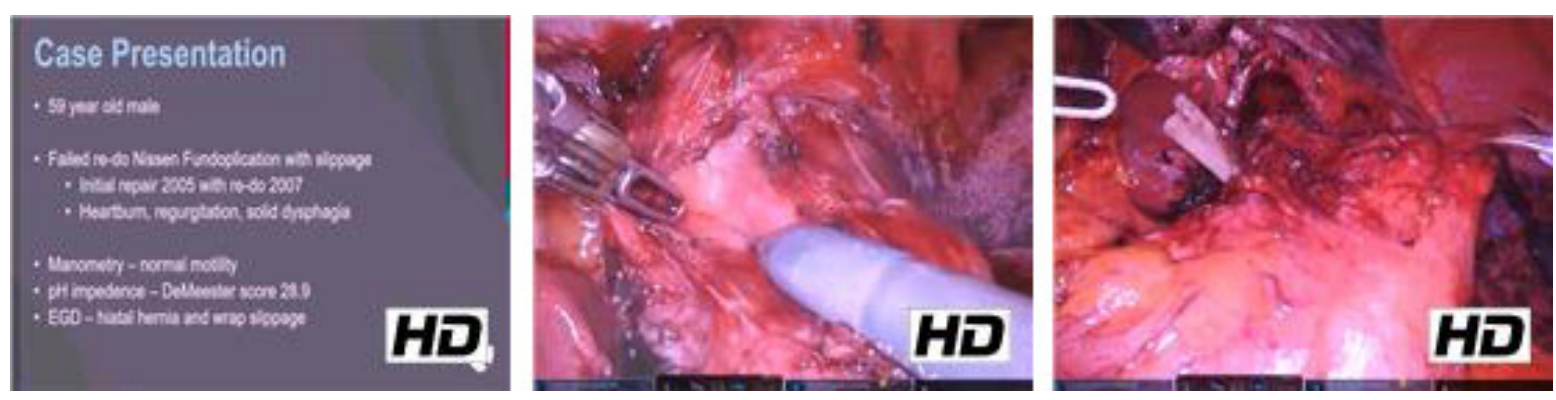

\section{Abstract}

Introduction: Recurrent hiatal hernia repair may be indicated in cases wherein symptoms are uncontrolled with medications coupled with an anatomic recurrence. ${ }^{1,2}$ Up to $15 \%$ of patients may develop a recurrent hiatal hernia on imaging at 1 year postoperatively, but only $10 \%$ of these patients are symptomatic. The size of the index hernia is an independent predictor of recurrence, and although mesh may decrease short-term recurrence, it has not been found to impact long-term recurrence rates. ${ }^{3}$ This video shows the case of a 59-year-old male, with a history of two prior hiatal hernia repairs with Nissen fundoplication. This video was first presented at the American College of Surgeons 2018 Clinical Congress in Boston, Massachusetts.

Methods: The patient presented to our institution with recurrent reflux symptoms including heartburn and regurgitation. Maximal nonoperative management failed to improve symptoms. A preoperative upper gastrointestinal (GI) swallow study and esophagogastroduodenoscopy demonstrated slippage of the fundoplication through the hiatus and recurrent reflux. His DeMeester score was $28 \mathrm{on} \mathrm{pH}$ impedance testing and his manometry was normal. We recommended a robotic redo hiatal hernia repair and Nissen fundoplication, with the possibility for a Collis gastroplasty. The patient was at risk for a gastroplasty secondary to the prior mediastinal dissection and esophagitis that may induce 
esophageal shortening. The gastroplasty would extend the length of his intra-abdominal esophagus and allow for a tension-free fundoplication. The reoperative hiatal hernia surgery started by defining the crural mechanism and dividing the attachments from the previous repair. The dissection extended into the mediastinum to the level of the pulmonary vessels. During take down of the Nissen fundoplication, the posterior border of the wrap was identified to avoid injuring the esophagus. Once the wrap was dissected from the esophagus, there was $<3 \mathrm{~cm}$ of intra-abdominal esophagus. A stapled Collis gastroplasty was then performed, excising a wedge of fundus and thereby lengthening the intra-abdominal esophagus before performing a symmetric Nissen fundoplication.

Results: The upper GI swallow study performed on postoperative day 1 demonstrated adequate neoesophageal length without a leak. The patient was discharged on postoperative day 2 tolerating a soft diet. He was recently seen at 1 year follow-up and is symptom free, with an upper Gl study demonstrating an intact subdiaphragmatic wrap.

Conclusion: In our experience, robotic surgery-with benefits of improved visualization and precisely controlled dissection - provides a safe and effective approach to challenging reoperative hiatal hernia repairs. The robot facilitates an extended dissection needs into the mediastinum and stapler angles that can be challenging for a wedge fundectomy.

No competing financial interests exist.

Runtime of video: 9 mins 33 secs

Funding Information: Funding for this study was provided by the Center for Advanced Surgical Technology at the University of Nebraska Medical Center.

This video was first presented as a video abstract at the American College of Surgeons 2018 Clinical Congress.

Keywords: hiatal hernia, paraesophageal hernia, Collis gastroplasty, Nissen fundoplication, redo

\section{Cite this video}

Salim Hosein, Dietric Hennings, Crystal Krause, Dmitry Oleynikov, Robotic Recurrent Hiatal Hernia Repair, Collis Gastroplasty, and Nissen Fundoplication, Videoscopy. 2019, DOI: 10.1089/vor.2019.0604.

\section{References}

1. Haider M, Iqbal A, Salinas V, Karu A, Mittal SK, Filipi CJ. Surgical repair of recurrent hiatal hernia. Hernia 2006;10:13-19.

2. Tolboom RC, Draaisma WA, Broeders IA. Evaluation of conventional laparoscopic versus robotassisted laparoscopic redo hiatal hernia and antireflux surgery: A cohort study. J Robot Surg 2016;10:33-39.

3. Armijo PR, Pokala B, Misfeldt M, Pagkratis S, Oleynikov D. Predictors of hiatal hernia recurrence after laparoscopic anti-reflux surgery with hiatal hernia repair: A prospective database analysis. J Gastrointest Surg 2019;23:696-701.

Original Publication Date: 2018 